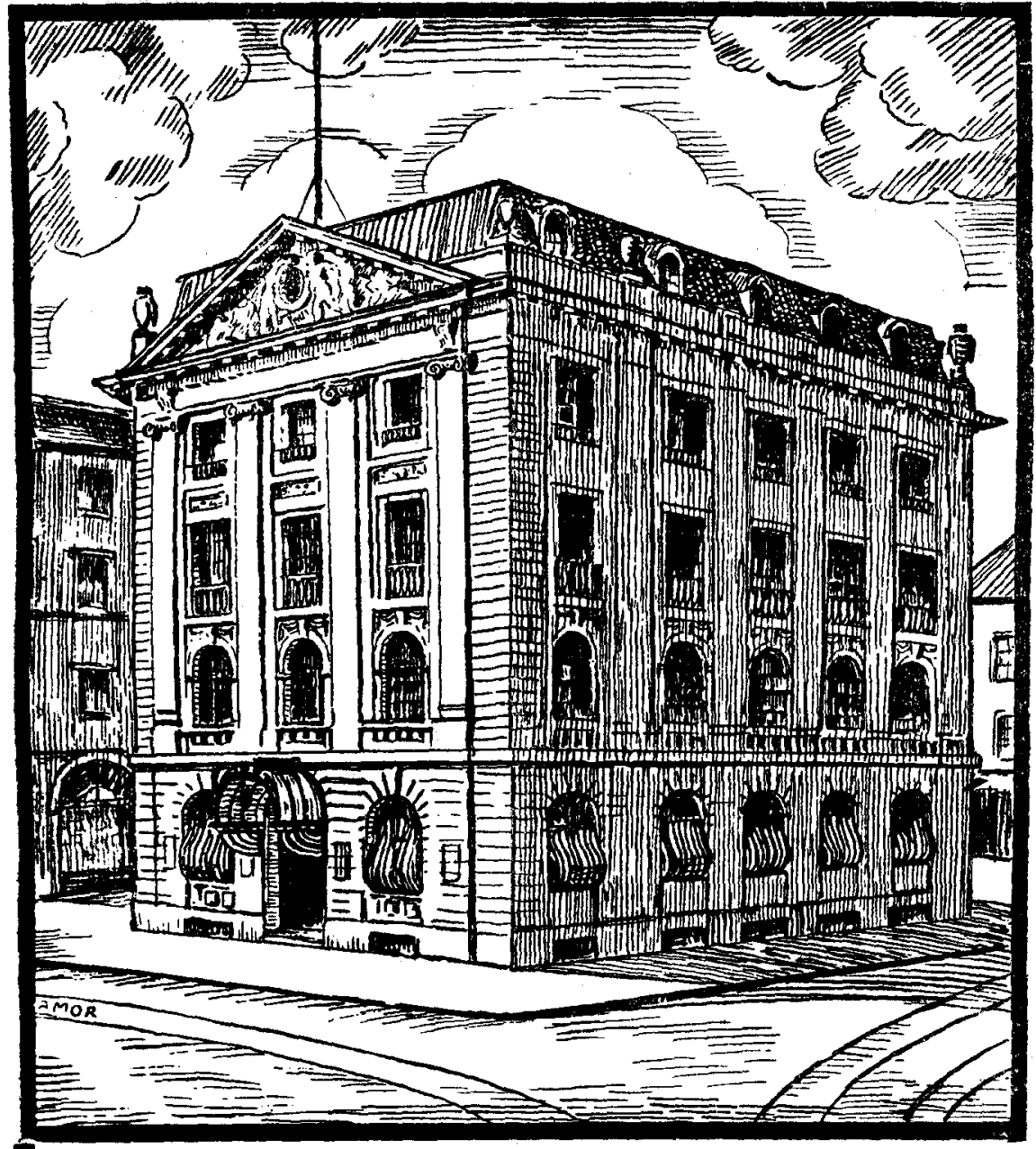

\title{
Société de
}

\section{BANQUE SUISSE}

Fondée en 1872

Capital-actions et réserves : 153 millions

\section{GENÈVE \\ 6-10, Corraterie}




\section{Vient de paraître}

\section{REVUE INTERNATIONALE DE L'ENFANT}

publiée par

L'UNION INTERNATIONALE DE SECOURS

AUX ENFANTS

31, Quai du Mont-Blanc, Genève (Suisse)

Le numéro (96 pages) : $1 \mathrm{fr}$. suisse. 12 numéro (un an): $10 \mathrm{fr}$. suisses. Publie des articles en cinq langues (français, anglais, allemand, italien, espagnol).

La Revue internationale de l'enfant est destinée à remplacer les trois périodiques de l'Union internationale de secours aux erifants: Bulletins en français et en anglais, et Salvate Parvulos (Bibliographie). Les informations qui formaient la matière de ces publications se retrouvent donc dans la nouvelle Revue. Elles sont précódées d'articles originaux, rédigés par les meilleurs spécialistes de tous pays - médecins, hygiénistes, juristes, sociologues - en cette science nouvelle qu'est la protection de l'enfance.

Figurent au sommaire du $5^{\text {me }}$ numéro :

Character Development through Recreation in Cities, by Dame Katharine Furse, G.B.E., London.

Les journaux d'enfants, par E. Clouzot, secrétaire général de I'Union internationale de secours aux enfants, Genève (illustré).

Die Lehrlingsf ürsorge in Oesterreich, von Anton Kimml, Referent für Lehrlingswesen bei der Wiener Kammer für Arbeiter und Angestellte.

Ein deutsches Gesetz gegen Schmutz- und Schundliteratur, von Frau Dr Elsa Matz, Mitglied des Deutschen Reichstags, Berlin.

Dans les Now suivants paraîtront des articles du Dr Franz Breunlich; de I. Heijermans, d'Amsterdam; de M. Jules Novagh, de. Budapest; de M. Andreas Boje, de Copenhague; de M. le Prof. Ad.. Czerny, de Berlin. 

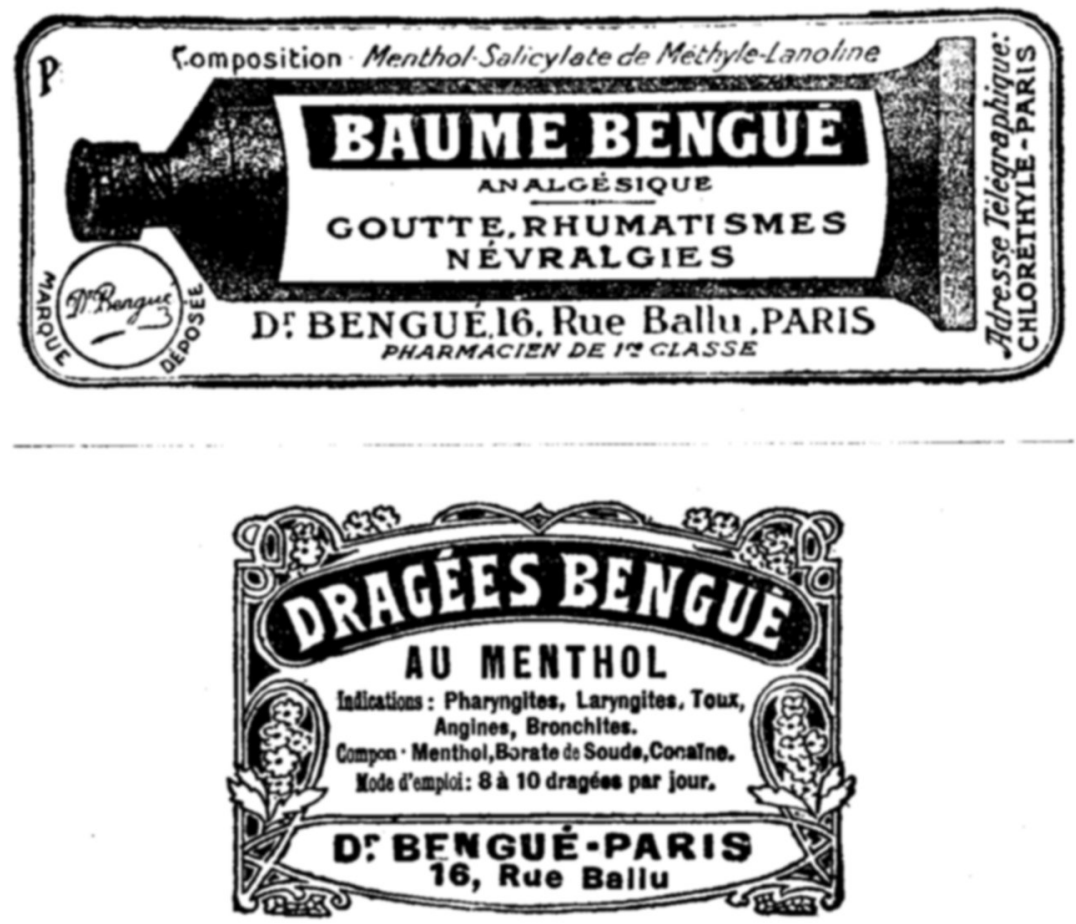

... à l'angle de la Rue du Rhône et de la Rue du Commerce

\section{L'UNION DE BANQUES SUISSES}

Capital et Réserves : 86 millions.

30 sièges et succursales.

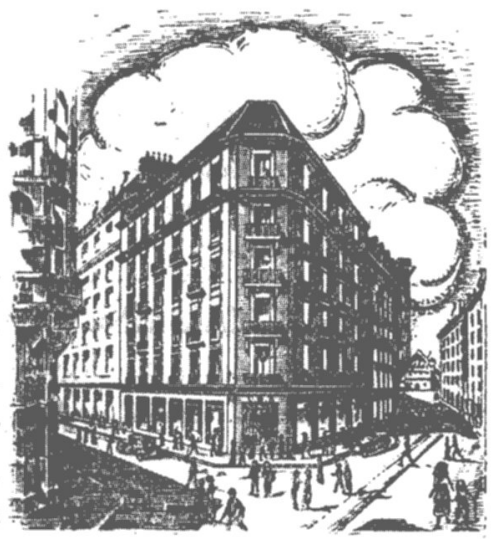

Assiste

de ses services et conseils Capitalistes et Commerçants.

\section{Facilite}

le règlement de leurs affaires en Suisse et à l'Etranger.

Renseignements au Secrétariat. 


\section{Banque Ottomane}

Capital Istg. 10.000.000. - dont moltie varsere.

Siège central à Constantinople, GALATA.

Agences à Péra et Stamboul, ainsi que dans les principales localités de la Turquie, l'Egypte, Chypre, la Palestine, la Mésopotamie, la Perse et la Grèce.

a Londres : 26, Throgmorton Street, E.C.2.,

d Manchester : 56/60, Cross Street, d Paris : 7, rue Meyerbeer ( $9^{\circ}$ Arr.), à Marseille : 41 et 43, rue Grignan, a Tunis : 68, Avenue Jules Ferry.

Se charge de toutes operations de Banque.

Filiale pour la Syrie : Banque de Syrie et du Grand Liban.

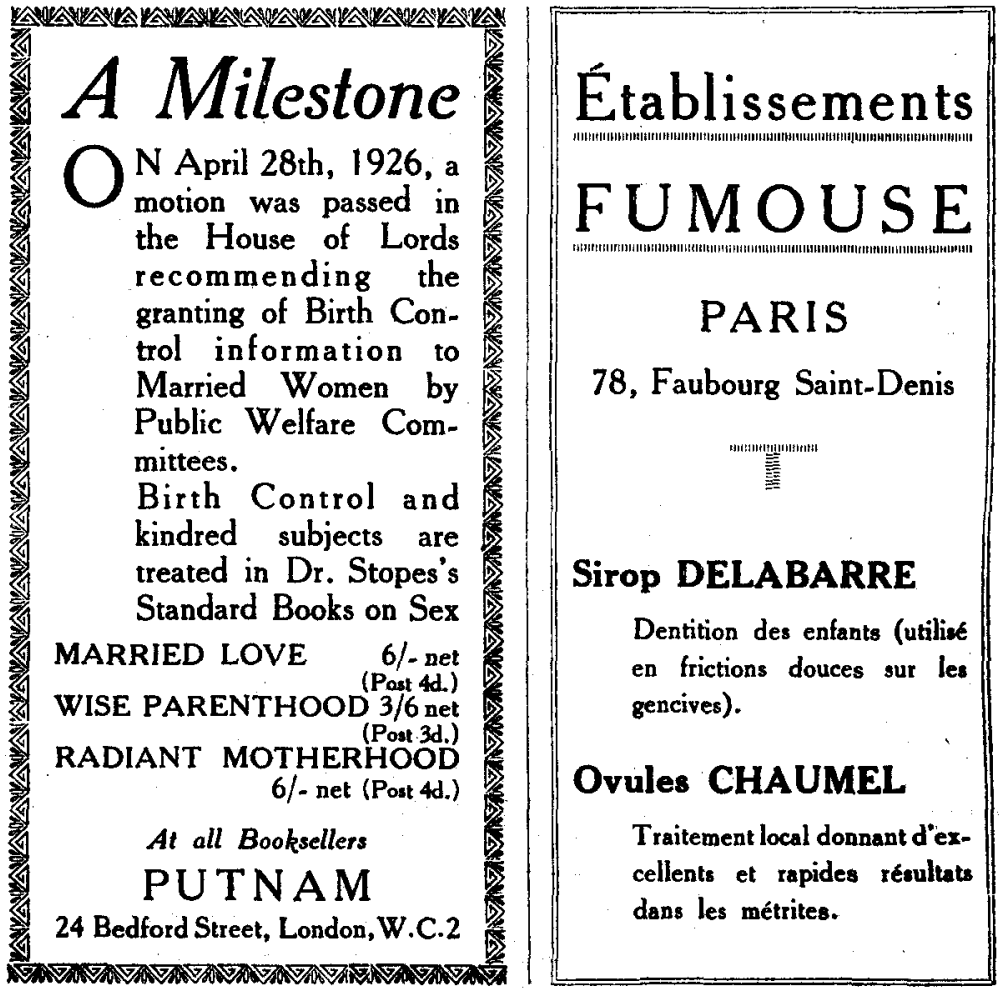




\section{Adresses des Comités centraux (suite).}

BELGIQUE. - Croix-Rouge de Belgique, rue de Livourne, 80, Bruxelles.

BOLIVIE. - Croix-Rouge bolivienne, Casilla de Correo, 504, Ia Paz.

BRESIL. - Croix-Rouge brésilienne, rue Utbaldino do Ámaral, 75, Rio de Janeiro.

BULGARIE. - Croix-Rouge Bulgare, 49, boulevard de Macédoine, Sofia.

CHILI. - Croix-Rouge chilienne, Correo, 8, Casilla 7577, Santiago de Chile.

CHINE. - Croix-Rouge chinoise, Pékin et 26, Kiukiang Road, Shanghaĩ.

COLOMBIE. - Croix-Rouge colombienne, Bogota.

COSTA-RICA. - Croix-Rouge costaricienne, Calle 5. Sur., San José.

CUBA. - Croix-Rouge cubaine, Ignacio Agramonte et av. de Belgica, La Havane.

DANEMARK. - Croix-Rouge danoise, Ved Stranden, 2, Copenhague, K.

DANTZIG. - Croix-Rouge de Dantzig, Neugarten, 12-16, Dantzig.

EGYPTE.- Croissant-Rouge égyptien, 21, Charea Gamea Sharkass, Le Caire.

EMPIRE BRITANNIQUE. - Croix-Rouge britannique (British Red Cross Society), Berkeley Street 19, London, W. 1.

AFRIQUE DU SUD. - Croix-Rouge sud-africaine, 6, 7 et 8 Bothwell House, Johannesburg. AUSTralID. - Croix-Ronge australienne, 42-46, Latrobe Street, Melbourne.

CaNADA. - Croix-Pouge canadionne, 410, Sherbourne Street, Toronto.

INDES ANGLaISTS. - Croix-Rouge des Indes Anglaises, Head-Quarters, Delhi.

Nod VELIE Z Źl.ANDE. - Croix-Rouge néo-zélandaise, P. O. Box 969, Wellington, N.Z.

EQUATEUR. - Croix-Rouge équatorienne, Vénézuela 46, Quito.

ESPAGNE. - Croix-Rouge espagnole, Calle de Sagâsta, 10, (Estafeta, 4), Madrid.

ESTHONIE. - Croix-Pouge esthonienne, Niguliste Tün, 12, Reval.

ETATS-UNIS. - Crolx-Rouge américaine (Central Committee of the American National Red Cross), Washington, D.C.

EINLANDE. - Croix-Rouge finlandaise, 19, Smedsgatan, Helsingfors.

FRANCE. - Croix-Rouge française, rue François ler, 21, Paris, 8me.

GRECE. - Croix-Rouge hellénique, rue Solon, 41, Athènes.

GUATEMALA. - Croix-Rouge guatémalienne, 13, Calle Oriente, 2, Guatémala.

HONGRIE. - Croix-Rouge hongroise, Andrassy-ut, 8, Budapest, IV.

ISLANDE. - Croix-Rouge islandaise, Reykjanik.

ITALIE. - Croix-Rouge italienne, via Toscana, 12, Rome.

JAPON. - Croix-Rouge japonaise, 5 Shiba Park, Tokio. (25).

LETTONIE. - Croix-Rouge lettone, Skolas ielá, 1, Riga.

LITHUANIE. - Croix-Rouge lithuanienne, Kestucio g-vé 8, Kaunas.

LUXFMBOURG. - Croix-Rouge luxembourgeoise, Luxembourg.

MEXIQUE. - Croix-Rouge mexicaine, 2, a de San Geronimo, 14, Mexico, D. F.

NORVEGE. - Croix-Rouge norvégienne, Akersgaten, 44, Oslo.

PANAMA. - Crojx-Rouge de Panama, Panama.

PARAGUAY. - Croix-Rouge paraguayenne, Avenida Espana, 505, Assomption.

PAYS-BAS. - Croix-Rouge néerlandaise, Princegsegracht, 27, La Haye.

INDFS NErRLANDAIses. - Croix-Rouge des Indes néerlandaises, Batavia.

PEROU. - Croix-Rouge péruvienne; Melchormalo 349, Lima.

PERSE. - Lion et Soleil-Rouges de Perse, Téhéran.

POLOGNE. - Croix-Rouge polonaise, rue Smolna, 6, Varsovie.

PORTUGAL. - Croix-Pouge portugaise, 1, Plaça do Commercio, Lisbonne.

ROUMANIE. - Croix-Rouge roumaine, rue Poincaré, 20, Bucarest.

SAN SALVADOR. - Croix-Rouge de San Salvador, 13 a Avenida Norte y 9 a calle Poniente, San Salvador.

SERBIE. - Crojx-Rouge des Serbes, Croates et Slovènes, Simina ulica, 21, Belgrade.

SIAM. - Croix-Rouge siamoise, Bangkok.

SUFDE. - Croix-Rouge suédoise (Svenska Roda Korset), Artillerigatan, 6, Stockholm, 14.

SUISSE. - Croix-Rouge suisse, Taubenstrasse, 8 , Berne.

TCHÉCOSLOVAQUIE. - Croix-Rouge tchécoslovaque, Neklanova ul., 147, Prague.

TURQUIE. - Croissant-Rouge turc, Angora.

U.R.S.S. (Union des républiques soviétiques socialistes.) - Alliance des sociétés de la

Croix-Pouge et du Croissant-Rouge de l'U.R.S.S., Moscou.

URUGUAY. - Croix-Rouge urugravenne, Bd Artigas 1938, Monterideo.

VENEZUELA. - Croix-Rouge vénézuélienne, Cuji a Punceres, 7, Caracas. 


\title{
Sommaire du Bulletin
}

\author{
T. LVII, $\mathbf{N}^{\circ} 286$ \\ JUIN 1926
}

Comité international. - Publication, 483.

Australie. - Assemblée générale annuelle de la Croix-Rouge australienne, 484 .

Canada. - Assemblée annuelle du Comité central de la CroixRouge, 486 .

Danemark. - 50me anniversaire de la Croix-Rouge, 489.

Indes britanniques. - La Croix-Rouge à Nagpur et à Mereara, 490. Japon. - Poème impérial (planche hors texte), 492.

Norvège. - Nouvelles de la Croix-Rouge, 492.

Paraguay. - Aetivité de la Croix-Rouge 1924-1925, 493. - Commission des dames de la Croix-Rouge paraguayenne, 494.

Serbie. - La Croix-Rouge et les réfugiés russes, 495.

Suède. - Rapport annuel, 496. - Publication, 497.

La Revue internationale de la Croix-Rouge, Bulletin international des Sociétés de la Croix-Rouge, parait à la fin de chaque mois.

Prix, franco, un an: Fr. 20.- Le numéro: Fr. 2.Années 1919, 1920 et 1921 (rares): Fr. 30.Administration : Promenade du Pin, 1, Genève. 